\title{
Choroidal thickness measurement by enhanced depth imaging and swept-source optical coherence tomography in central serous chorioretinopathy
}

\author{
Ferdiriva Hamzah ${ }^{1,2+}$, Ari Shinojima ${ }^{2 *}$, Ryusaburo Mori ${ }^{2+}$ and Mitsuko Yuzawa ${ }^{2+}$
}

\begin{abstract}
Background: We evaluated subfoveal choroidal thickness measured with two different forms of optical coherence tomography (OCT), enhanced-depth imaging (EDI) and swept-source (SS) OCT, in central serous chorioretinopathy (CSC).

Methods: Fifty-six eyes of 48 patients diagnosed with acute or chronic CSC, were studied prospectively. Subfoveal choroidal thickness was measured as the distance between the outer border of the retinal pigment epithelium-Bruch's membrane complex, and the chorioscleral border under the fovea. Subfoveal choroidal thickness was measured using EDI-OCT and SS-OCT. We also measured serous retinal detachment (SRD) only with SS-OCT. The Pearson correlation coefficient was used to assess the correlation between subfoveal choroidal thickness values determined by the two different OCT modalities.

Results: The mean patient age was $52 \pm 13$ years (range, 32-82 years). Among the 56 eyes, 21 had acute CSC and 35 had chronic CSC. Subfoveal choroidal thickness measured with EDI-OCT was $336.6 \pm 91.6 \mu \mathrm{m}$ in acute and $388.0 \pm 103.4 \mu \mathrm{m}$ in chronic CSC. With SS-OCT, the thickness in acute CSC was $332.0 \pm 96.7 \mu \mathrm{m}$ and that in chronic CSC was $392.6 \pm 101.3 \mu \mathrm{m}$. Acute CSC ( $p<0.001$, correlation coefficient; $r=0.99)$ and chronic CSC $(p<0.001$, correlation coefficient; $r=0.97$ ) values obtained with the two different $O C T$ modalities correlated significantly. Among the 56 eyes, 43 (19 eyes with acute and 24 with chronic CSC) were evaluable for SRD height by SS-OCT. The mean SRD height was $128.9 \pm 83.6 \mu \mathrm{m}$ in acute cases and $96.3 \pm 62.0 \mu \mathrm{m}$ in chronic cases.
\end{abstract}

Conclusions: Subfoveal choroidal thickness obtained with two different OCT modalities correlated significantly.

\section{Background}

Choroidal abnormalities are associated with the pathogenesis of various disorders affecting the retina including central serous chorioretinopathy (CSC) [1,2].

In CSC patients, the choroid is thicker than in normal eyes. Choroidal vascular hyper-permeability is frequently seen in eyes with CSC. This raises hydrostatic pressure in the choroid and thereby increases choroidal thickness $[3,4]$.

In recent years, detecting the choroid using spectraldomain optical coherence tomography (SD-OCT) has

\footnotetext{
*Correspondence: ariearly7@gmail.com

${ }^{\dagger}$ Equal contributors

2Jakarta Eye Center, Jakarta, Indonesia

Full list of author information is available at the end of the article
}

often been difficult, because the retinal pigment epithelium (RPE) hinders the penetration process. This is because the wavelength of the light source is occasionally not long enough to penetrate into the choroid. Conventional OCT employs a wavelength of about $800 \mathrm{~nm}$, while the wavelengths capable of clear choroidal imaging are reportedly in the $1,060 \mathrm{~nm}$ range [5-7].

Enhanced depth imaging (EDI) OCT is a recent modification of the standard technique. This novel modality shows the cross-sectional structure and thickness of the choroid using commercial SD-OCTs. Basically, the EDIOCT modality places the objective lens of the SD-OCT closer to the eye, such that the light backscattered from the choroid is closer to the zero-delay and sensitivity is 
thereby enhanced. Therefore, this modality produces better imaging of the choroid $[8,9]$.

However, even though EDI-OCT can enhance the sensitivity of the choroid, light scattering by the RPE and choroid remains a problem. In this event, the chorioscleral border cannot be detected, especially when a subject happens to have a particularly thick choroid. Measurable choroidal thickness has been reported in $95.8 \%$ of the eyes examined by Heidelberg EDI-OCT, while thickness cannot be determined in the remainder due to poor image quality obscuring the outer boundary of the choroid [10]. Yamashita et al. reported that choroidal thickness measurements were unsuccessful in some healthy eyes examined (4 out of 43 eyes) because the subjects had choroidal thicknesses exceeding $500 \mu \mathrm{m}$, making it impossible to clearly see the outer boundary of the choroid [11].

Topcon Deep Range Imaging OCT is another new technique designed to produce better imaging of the choroid. It has a scanning speed of 100,000 A-scans/second and is based on Swept-Source (SS) technology. It utilizes a wavelength of $1,050 \mathrm{~nm}$ and thereby provides deeper penetration into the choroid with higher image quality. The longer the wavelength of the light, the less is the scattering reflection of the RPE, thus enabling deep and full-thickness choroidal imaging even up to the scleral surface. SS-OCT showed a clearly defined, measurable posterior portion of the choroid [5-7,12].

OCT has now been proven to be an effective noninvasive tool for evaluating and detecting choroidal changes in pathological states [13-15]. It is also important to evaluate and compare the measurements of choroidal thickness obtained using different instruments on subjects with pathological conditions. Moreover, SS-OCT can be used in addition to choroidal imaging in clinical practice $[7,13]$.

\section{Methods}

Between November 2012 and September 2013, we studied 56 eyes of 48 CSC patients (41 men, 49 eyes; 7 women, 7 eyes) at Surugadai Nihon University Hospital, Tokyo, Japan. This study was approved by the institutional review board of Surugadai Nihon University Hospital and written informed concent was obtained from all patients. All subjects underwent a comprehensive ophthalmic examination, that included a thorough ocular examination using an indirect ophthalmoscope and slit-lamp biomicroscope with a contact lens, including an autorefractometer, bestcorrected visual acuity (BCVA) measurement with the Landolt $\mathrm{C}$ eye chart, color fundus photography (TRC, 50IX/Imagenet, Topcon, Tokyo, Japan), intraocular pressure measurement, slit-lamp examination, dilated funduscopy, fluorescein angiography (FA), indocyanine green angiography, EDI-OCT and SS-OCT. CSC was objectively diagnosed based on the angiographic findings. If both eyes met the inclusion criteria, then the subfoveal choroidal thickness was measured regardless of whether the case was unilateral or bilateral, and the value was included in this study. Acute CSC was defined as detachment of the neurosensory retina which became symptomatic within 6 months. Chronic CSC was defined as detachment of the neurosensory retina in terms of symptoms, which persisted for at least 6 months. All subjects were imaged with Heidelberg Spectralis (Heidelberg Engineering Inc., Heidelberg, Germany) using the EDI-OCT technique and Topcon Deep Range Imaging (DRI) SS-OCT (Topcon Corp, Tokyo, Japan) on the same day around noon to avoid diurnal variations [16]. A horizontal section through the fovea was obtained for measurement. The scan was performed with each instrument using a single horizontal scan line. The length of the scan line was $12 \mathrm{~mm}$ in SSOCT, $6 \mathrm{~mm}$ in EDI-OCT. There were 100,000 A-scans/ second per line in SS-OCT, and 40,000 A-scans/second per line in EDI-OCT. Both instruments were equipped with an eye-tracking modality.

The subfoveal choroidal thickness was measured as the distance between the outer border of the RPE-Bruch's membrane complex and the chorioscleral border under the fovea. It was measured manually using the caliper tool. If the line was blurred at the chorioscleral interface, the center of the traceable line was measured. We measured serous retinal detachment (SRD) only with SS-OCT, from the outer surface of the neurosensory retina and the RPE. Cases with a myopic refractive error exceeding 6.0 Diopters, a history of intraocular surgery, photodynamic therapy, other intraocular diseases including retinal dystrophies, uveitis, macular hole, epiretinal membrane, and/ or macular edema not associated with CSC, were excluded from this study. Patients who had undergone focal laser photocoagulation were included in the study $[3,4]$.

All data were expressed as means \pm standard deviation (SD). The Pearson correlation coefficient was used to assess the correlation between subfoveal choroidal thickness values determined by the two different OCT modalities. Statistical analysis was performed using SPSS software (Version 21.0 for Windows, IBM-SPSS, Japan).

\section{Results}

The mean age of the patients was $52 \pm 13$ years (range, $32-82$ years). The mean refractive error was $-0.1 \pm 1.9 \mathrm{Di}$ opters (mean $\pm \mathrm{SD}$, range, $-5.5-3.75$ Diopters). Among the 56 eyes, 21 had acute CSC and 35 had chronic CSC. The mean BCVA was $1.1 \pm 0.4$ decimals (mean $\pm \mathrm{SD}$, range, $0.2-1.5)$. Mean intraocular pressure was $15.4 \pm 2.7 \mathrm{mmHg}$ (mean \pm SD, range, 9-21 $\mathrm{mmHg}$ ). The duration of acute CSC from the onset was $7.4 \pm 6.6$ weeks (mean \pm SD, range, 1-20 weeks), and the duration of chronic CSC from the onset was $62.1 \pm 51.5$ weeks (mean \pm SD, range, 26260 weeks). Good quality B-scan images from EDI- and 
SS-OCT (Figure 1) were obtained for all eyes. With SS-OCT, the mean SRD heights in acute and chronic CSC cases were 128.9 $\pm 83.6 \mu \mathrm{m}$ and $96.3 \pm 62.0 \mu \mathrm{m}$, respectively.

Subfoveal choroidal thickness in acute CSC measured with EDI-OCT was $336.6 \pm 91.6 \mu \mathrm{m}$ (mean $\pm \mathrm{SD}$, range, 225-534 $\mu \mathrm{m}$ ) and that in chronic CSC was $388.0 \pm$ 103.4 $\mu \mathrm{m}$ (mean $\pm \mathrm{SD}$, range, 170-587 $\mu \mathrm{m}$ ). With SS-OCT, the thickness in acute CSC was $332.0 \pm 96.7 \mu$ m (mean \pm $\mathrm{SD}$, range, 212-532 $\mu \mathrm{m})$ and that in chronic CSC was $392.6 \pm 101.3 \mu \mathrm{m}$ (mean $\pm \mathrm{SD}$, range, $190-573 \mu \mathrm{m})$. Choroidal thickness values obtained with the two different OCT modalities showed a significant correlation. Acute CSC $(\mathrm{p}<0.001$, correlation coefficient; $\mathrm{r}=0.99)$ and chronic CSC ( $p<0.001$, correlation coefficient; $r=0.97$ ) values are presented in Figure 2.

\section{Discussion}

EDI is a recently standardized technique for imaging the choroid more clearly using commercial SD-OCT [17-20]. Yamashita et al. reported that $90.7 \%$ of subjects had measurable choroidal thickness when examined using three different SD-OCT modalities, including EDI-OCT [11].

Topcon DRI is another new form of OCT that provides higher penetration enabling deep choroidal imaging. Moreno et al. reported $100 \%$ of the definite chorioscleral junction to be visualized with SS-OCT (DRI) [5]. The SS-OCT facilitated visualization for reliable thickness measurements. The EDI-OCT occasionally requires the contrast to be set in order to trace the chorioscleral borders clearly, especially in patients with a subfoveal choroidal thickness exceeding $500 \mu \mathrm{m}$. No eyes were excluded from our study due to poor-quality EDI-OCT images. Occasionally, it was necessary to change the image contrast, but this allowed us to detect the chorioscleral border in all eyes, even those with a maximum thickness of $587 \mu \mathrm{m}$ on EDI-OCT, and a maximum thickness of $573 \mu \mathrm{m}$ on SS-OCT.

Normal subfoveal choroidal thickness varies widely among reports [7,8,21]. Ikuno et al. [7] found that the choroidal thickness values in healthy eyes correlated well between the $1,060 \mathrm{~nm}$ and $870 \mathrm{~nm}$ OCT instruments,

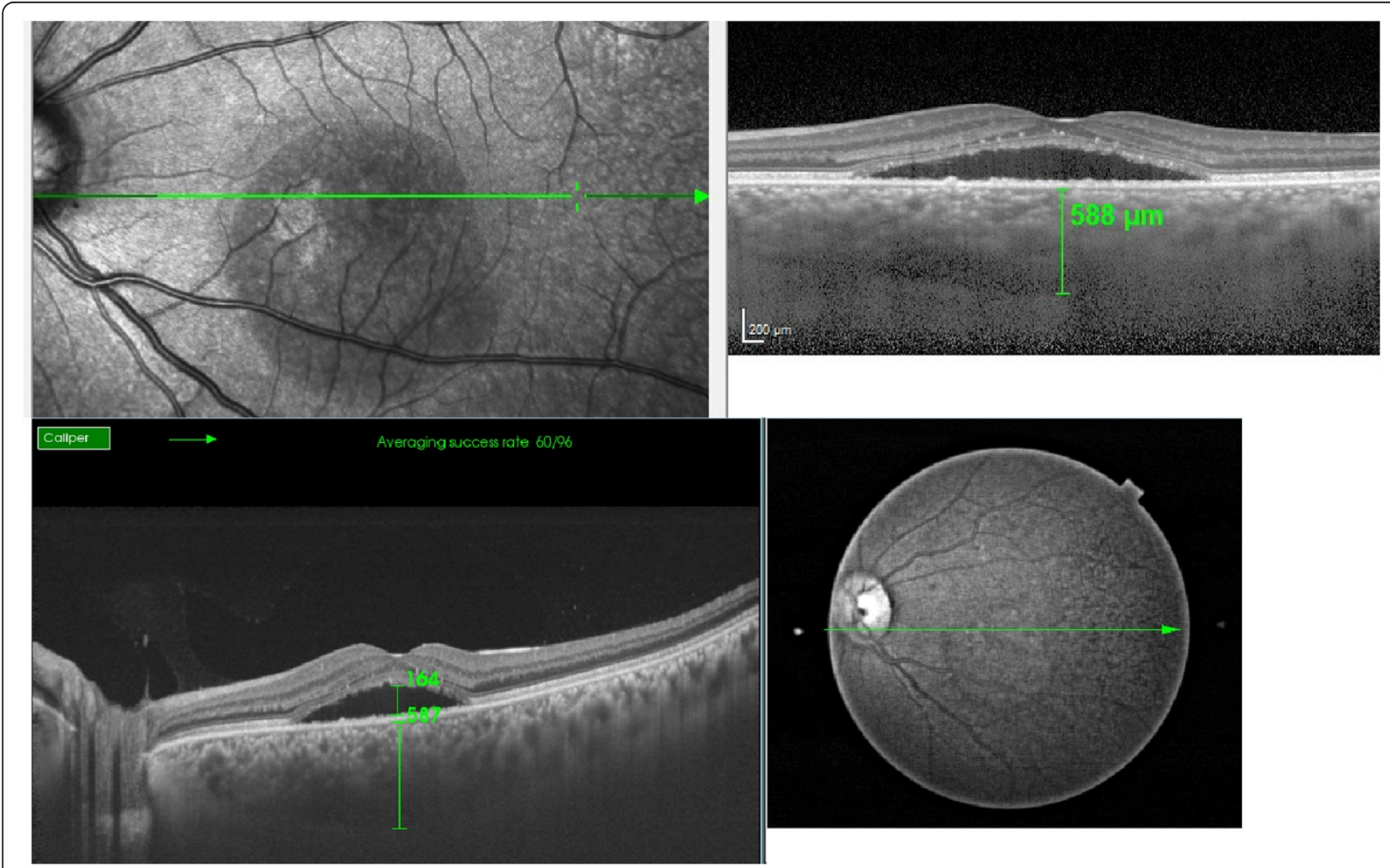

Figure 1 Representative images obtained with two different forms of optical coherence tomography. Chronic unilateral central serous chorioretinopathy, in the left eye, with onset 12 months prior to study enrollment. Enhanced depth imaging optical coherence tomography (EDI-OCT) (Top). Swept-source OCT (SS-OCT) image (Bottom). Both images were obtained on the same day. The subfoveal choroidal thickness was measured as the distance between the outer border of the retinal pigment epithelium-Bruch's membrane complex and the chorioscleral border under the fovea. Subfoveal choroidal thickness was $588 \mu \mathrm{m}$ in EDI-OCT (Top), and $587 \mu \mathrm{m}$ in SS-OCT (Bottom). Serous retinal detachment was measured between the outer surface of the neurosensory retina and the retinal pigment epithelium, and the height was $164 \mu \mathrm{m}$ in SS-OCT (Bottom). 

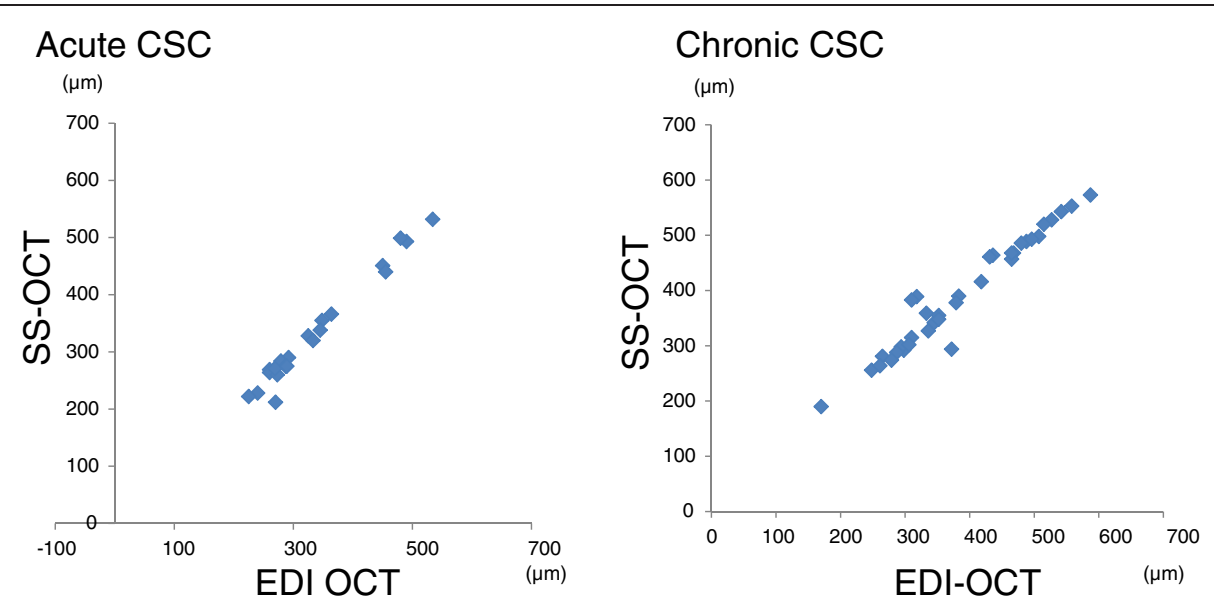

Figure $\mathbf{2}$ Correlation between choroidal thickness values measured with two different forms of optical coherence tomography. Both figures show the linear correlation between enhanced depth imaging optical coherence tomography (EDI-OCT) and swept-source OCT. Acute central serous chorioretinopathy (CSC) $(n=21$, correlation coefficient; $r=0.99 ; p<0.001)$ (Left figure). Chronic CSC ( $n=35$, correlation coefficient; $r=0.97 ; p<0.001)$ (Right figure).

but their report did not include eyes with pathological conditions.

In our study, even in symptomatic eyes with subretinal fluid, the choroidal thickness values obtained with two different OCT modalities showed a significant correlation. This was true for both acute CSC $(\mathrm{p}<0.001$, correlation coefficient; $r=0.99)$ and chronic CSC $(\mathrm{p}<0.001$, correlation coefficient; $r=0.97$ ).

Imamura et al. [9] reported the subfoveal choroidal thickness measured with EDI-OCT in CSC to be greater than that in normal eyes. Maruko et al. [4] reported choroidal thickness, as measured with EDI-OCT, to be $344 \pm 112 \mu \mathrm{m}$ in acute CSC and $360 \pm 123 \mu \mathrm{m}$ in chronic CSC. Ferrara et al. [22] reported choroidal thickness measured with SS-OCT to be $351 \pm 84 \mu \mathrm{m}$ in chronic CSC. Compared with normal eyes, subfoveal choroidal thickness was significantly increased in both the eyes with active CSC and in the unaffected fellow eyes $(\mathrm{P}<0.001$ in both groups) [23].

In this study, chronic CSC was also associated with a slightly thicker choroid than acute CSC. Increased hydrostatic pressure develops within the choroid of CSC patients and it is possible that the choroidal thickness is thereby altered.

Negi and Marmor demonstrated in their experimental studies that focal damage, such as that produced by photocoagulation, appears to facilitate water movement from, rather than into, the subretinal space [24]. RPE damage in chronic CSC and photocoagulation scar findings appear to correspond to window defects, especially those observed on FA. Chronic CSC is associated with extensive RPE damage in the macular area, suggesting slightly more rapid resorption than in acute CSC. Therefore, subretinal fluid presumably flows toward the choroid and might thereby cause choroidal thickening through accumulation of fluid. This may be one of the factors leading to a thicker choroid in chronic as opposed to acute CSC cases. Ferrara et al. [22] reported that En face SS-OCT imaging at the level of the choriocapillaris showed hypoechoic spots suggesting the presence of enlarged vessels at this level in 8 (53\%) of the eyes in their study.

To summarize the characteristics of CSC in a large number of publications [25], the cut-off value for the height of SRD, acute vs chronic CSC cases, is $100 \mu \mathrm{m}$, i.e., acute CSC $>100 \mu \mathrm{m}$. In our study as well, with SS-OCT, the mean SRD heights in acute and chronic CSC cases were $128.9 \pm 83.6 \mu \mathrm{m}$ and $96.3 \pm 62.0 \mu \mathrm{m}$, respectively.

This study has limitations, including manual measurement of subfoveal choroidal thickness on both EDI-OCT and SS-OCT (DRI) and the small sample size. Automatic detection and segmentation systems are still lacking for the choroid.

\section{Conclusion}

Measuring choroidal thickness has become a tool facilitating the diagnosis of CSC. EDI-OCT and SS-OCT are useful for evaluating subclinical choroidal abnormalities in CSC and this study confirmed a correlation between the values obtained with these two OCT modalities.

\section{Competing interests}

The authors declare that they have no competing interests.

\section{Authors' contributions}

FH conceived this study, and participated in its design and implementation. AS helped to draft the manuscript and participated in the statistical analysis. RM critically revised the manuscript for important intellectual content. MY gave final approval of the version to be published. All authors have read and approved the final manuscript. 


\section{Acknowledgements}

We thank Bierta Barfod for English proofreading.

This study was funded in part by the Research Committee on Chorioretinal Degenerations and Optic Atrophy, The Ministry of Health and Welfare of Japan (Mitsuko Yuzawa). Neither the sponsor nor any other funding organization had a role in the design or conduct of this research.

\section{Author details}

'Department of Ophthalmology, School of Medicine, Surugadai Hospital of Nihon University, 1-8-13 Surugadai, Kanda, Chiyodaku, Tokyo 101-8309, Japan. ${ }^{2}$ Jakarta Eye Center, Jakarta, Indonesia.

Received: 13 May 2014 Accepted: 19 November 2014 Published: 25 November 2014

\section{References}

1. Sasahara M, Tsujikawa A, Musashi K, Gotoh N, Otani A, Mandai M, Yoshimura $\mathrm{N}$ : Polypoidal choroidal vasculopathy with choroidal vascular hyperpermeability. Am J Ophthalmol 2006, 142:601-607.

2. Jirarattanasopa P, Ooto S, Nakata I, Tsujikawa A, Yamashiro K, Oishi A, Yoshimura N: Choroidal thickness, vascular hyperpermeability, and complement factor $\mathrm{H}$ in age-related macular degeneration and polypoidal choroidal vasculopathy. Invest Ophthalmol Vis Sci 2012, 14:3663-3672.

3. Jirarattanasopa P, Ooto S, Tsujikawa A, Yamashiro K, Hangai M, Hirata M, Matsumoto A, Yoshimura N: Assessment of macular choroidal thickness by optical coherence tomography and angiographic changes in central serous chorioretinopathy. Ophthalmology 2012, 119:1666-1678.

4. Maruko I, lida T, Sugano Y, Ojima A, Sekiryu T: Subfoveal choroidal thickness in fellow eyes of patients with central serous chorioretinopathy. Retina 2011, 31:1603-1608.

5. Ruiz-Moreno JM, Flores-Moreno I, Lugo F, Ruiz-Medrano J, Montero JA, Akiba M: Macular choroidal thickness in normal pediatric population measured by swept-source optical coherence tomography. Invest Ophthalmol Vis Sci 2013, 54:353-359.

6. Hirata M, Tsujikawa A, Matsumoto A, Hangai M, Ooto S, Yamashiro K, Akiba M, Yoshimura N: Macular choroidal thickness and volume in normal subjects measured by swept-source optical coherence tomography. Invest Ophthalmol Vis Sci 2011, 52:4971-4978.

7. Ikuno Y, Maruko I, Yasuno Y, Miura M, Sekiryu T, Nishida K, lida T: Reproducibility of retinal and choroidal thickness measurements in enhanced depth imaging and high-penetration optical coherence tomography. Invest Ophthalmol Vis Sci 2011, 52:5536-5540.

8. Margolis R, Spaide RF: A pilot study of enhanced depth imaging optical coherence tomography of the choroid in normal eyes. Am J Ophthalmol 2009, 147:811-815.

9. Imamura Y, Fujiwara T, Margolis R, Spaide RF: Enhanced depth imaging optical coherence tomography of the choroid in central serous chorioretinopathy. Retina 2009, 29:1469-1473.

10. Mwanza JC, Hochberg JT, Banitt MR, Feuer WJ, Budenz DL: Lack of association between glaucoma and macular choroidal thickness measured with enhanced depth-imaging optical coherence tomography. Invest Ophthalmol Vis Sci 2011, 52:3430-3435.

11. Yamashita T, Shirasawa M, Arimura N, Terasaki H, Sakamoto T: Repeatability and reproducibility of subfoveal choroidal thickness in normal eyes of Japanese using different SD-OCT devices. Invest Ophthalmol Vis Sci 2012, 53:1102-1107.

12. Yasuno Y, Miura M, Kawana K, Makita S, Sato M, Okamoto F, Yamanari M, Iwasaki T, Yatagai T, Oshika T: Visualization of sub-retinal pigment epithelium morphologies of exudative macular diseases by high-penetration optical coherence tomography. Invest Ophthalmol Vis Sci 2009, 50:405-413.

13. Branchini L, Regatieri CV, Flores-Moreno I, Baumann B, Fujimoto JG, Duker JS: Reproducibility of choroidal thickness measurements across three spectral domain optical coherence tomography systems. Ophthalmology 2012, 119:119-123.

14. Regatieri CV, Branchini L, Fujimoto JG, Duker JS: Choroidal imaging using spectral-domain optical coherence tomography. Retina 2012, 32:865-876

15. Agawa T, Miura M, Ikuno Y, Makita S, Fabritius T, Iwasaki T, Goto H, Nishida K, Yasuno Y: Choroidal thickness measurement in healthy Japanese subjects by three-dimensional high-penetration optical coherence tomography. Graefes Arch Clin Exp Ophthalmol 2011, 249:1485-1492.
16. Brown JS, Flitcroft DI, Ying GS, Francis EL, Schmid GF, Quinn GE, Stone RA: In vivo human choroidal thickness measurements: evidence for diurnal fluctuations. Invest Ophthalmol Vis Sci 2009, 50:5-12.

17. Wong IY, Koizumi $H$, Lai WW: Enhanced depth imaging optical coherence tomography. Ophthalmic Surg Lasers Imaging 2011, 42:S75-S84.

18. Spaide RF, Koizumi H, Pozonni MC: Enhanced depth imaging spectraldomain optical coherence tomography. Am J Ophthalmol 2008, 146:496-500.

19. Kim SW, Oh J, Kwon SS, Yoo J, Huh K: Comparison of choroidal thickness among patients with healthy eyes, early age-related maculopathy, neovascular age-related macular degeneration, central serous chorioretinopathy, and polypoidal choroidal vasculopathy. Retina 2011, 31:1904-1911.

20. Maruko I, lida T, Sugano Y, Ojima A, Ogasawara M, Spaide RF: Subfoveal choroidal thickness after treatment of central serous chorioretinopathy. Ophthalmology 2010, 17:1792-1799.

21. Ikuno $Y$, Kawaguchi $K$, Nouchi T, Yasuno Y: Choroidal thickness in healthy Japanese subjects. Invest Ophthalmol Vis Sci 2010, 51:2173-2176.

22. Ferrara D, Mohler KJ, Waheed N, Adhi M, Liu JJ, Grulkowski I, Kraus MF, Baumal C, Hornegger J, Fujimoto JG, Duker JS: En face enhanced-depth swept-source optical coherence tomography features of chronic central serous chorioretinopathy. Ophthalmology 2014, 121:719-726.

23. Kim YT, Kang SW, Bai KH: Choroidal thickness in both eyes of patients with unilaterally active central serous chorioretinopathy. Eye (Lond) 2011, 25:1635-1640.

24. Negi A, Marmor MF: Experimental serous retinal detachment and focal pigment epithelial damage. Arch Ophthalmol 1984, 102:445-449.

25. Wang M, Munch IC, Hasler PW, Prünte C, Larsen M: Central serous chorioretinopathy. Acta Ophthalmol 2008, 86:126-145.

doi:10.1186/1471-2415-14-145

Cite this article as: Hamzah et al:: Choroidal thickness measurement by enhanced depth imaging and swept-source optical coherence tomography in central serous chorioretinopathy. BMC Ophthalmology 2014 14:145.

\section{Submit your next manuscript to BioMed Central and take full advantage of:}

- Convenient online submission

- Thorough peer review

- No space constraints or color figure charges

- Immediate publication on acceptance

- Inclusion in PubMed, CAS, Scopus and Google Scholar

- Research which is freely available for redistribution 